\title{
Schouten and Vrănceanu Connections on Golden Manifolds
}

\author{
Mustafa Gök*, Sadık Keleş and Erol Kılıç \\ (Communicated by Mukut Mani Tripathi)
}

\begin{abstract}
In this paper, we study two special linear connections, which are called Schouten and Vrănceanu connections, defined by an arbitrary fixed linear connection on a differentiable manifold admitting a golden structure. The golden structure defines two naturally complementary projection operators splitting the tangent bundle into two complementary parts, so there are two globally complementary distributions of the tangent bundle. We examine the conditions of parallelism for both of the distributions with respect to the fixed linear connection under the assumption that it is either the Levi-Civita connection or is not. We investigate the concepts of half parallelism and anti half parallelism for each of the distributions with respect to the Schouten and Vrănceanu connections. We research integrability conditions of the golden structure and its associated distributions from the viewpoint of the Schouten and Vrănceanu connections. Finally, we analyze the notion of geodesicity on golden manifolds in terms of the Schouten and Vrănceanu connections.
\end{abstract}

Keywords: golden structure; Schouten connection; Vrănceanu connection; parallelism; half parallelism; anti half parallelism; integrability; geodesic. AMS Subject Classification (2010): Primary: 53C05; 53C15; Secondary: 58A30; 53C22; 53C25.

\section{Introduction}

In [1], S. I. Goldberg and K. Yano have introduced the notion of a polynomial structure of degree $n$ on a $C^{\infty}$-differentiable manifold $M$ as a $C^{\infty}$-tensor field $f$ of type $(1,1)$ satisfying the algebraic equation

$$
Q(x)=x^{n}+a_{n} x^{n-1}+\cdots+a_{2} x+a_{1} I=0,
$$

where $I$ is the identity $(1,1)$-tensor field on $M$ and $f^{n-1}(p), f^{n-2}(p), \ldots, f(p), I$ are linearly independent for every point $p \in M$. The polynomial $Q(x)$ is called the structure polynomial. For example, under suitable conditions from the point of the dimension of $M$, an almost complex structure on $M$ is a polynomial structure of degree 2, an almost contact structure on $M$ is a polynomial structure of degree 3 and an almost product structure on $M$ is a polynomial structure of degree 2 . Therefore, the structure polynomial is a useful tool that continually generates new geometrical structures on differentiable manifolds of class $C^{\infty}$. By using it, C. E. Hreţcanu has constructed a new structure on a $C^{\infty}$-differentiable real manifold, called a golden structure, in order to research the effect of the golden ratio on differential geometry in [2]. In the last decade, it has become a subject of growing interest and been studied by many geometers.

A golden structure $\Phi$ on a $C^{\infty}$-differentiable real manifold $M$ is a tensor field of type $(1,1)$ satisfying the equation

$$
\Phi^{2}=\Phi+I,
$$

where $I$ is the identity $(1,1)$-tensor field on $M$. That is, the golden structure $\Phi$ is a polynomial structure of degree 2 with the structure polynomial $Q(x)=x^{2}-x-1$. The pair $(M, \Phi)$ is called a golden manifold $[2,3,4,5]$. A research of the geometry of golden structures has been initiated in [3]. Some applications of the golden ratio in differential geometry have been found by using an almost product structure on a $C^{\infty}$-differentiable real manifold. Basic properties, integrability and parallelism conditions, some examples of 
golden structures and treatment of connections in principal and tangent bundle in terms of golden structures have been examined.

In $[3,4,5]$, the concept of a golden Riemannian manifold has been defined as follows: A golden Riemannian manifold is a triple $(M, g, \Phi)$, where $M$ is a differentiable manifold of class $C^{\infty}, g$ is a Riemannian metric and $\Phi$ is a golden structure on $M$ such that $g$ and $\Phi$ satisfying the relation

$$
g(\Phi X, Y)=g(X, \Phi Y)
$$

for any vector fields $X, Y \in \Gamma(T M)$. Some fundamental properties and interesting results on golden Riemannian manifolds have been obtained in $[3,5]$.

Submanifolds of golden Riemannian manifolds have been studied by M. C. Crâşmăreanu and C. E. Hrețcanu in $[4,5]$. The authors have established several properties of the induced structure on any submanifold in a golden Riemannian manifold. Particularly, invariant submanifolds of a golden Riemannian manifold have been investigated. It has been proved that any invariant submanifold of a golden Riemannian manifold is also a golden Riemannian manifold. Moreover, it has been shown that the Nijenhuis tensor of the induced structure vanishes identically on invariant submanifolds in the event that the ambient manifold is a locally decomposable golden Riemannian manifold.

The main aim of this paper is to investigate the Schouten and Vrănceanu connections, defined by an arbitrary fixed linear connection on a $C^{\infty}$-differentiable real manifold admitting a golden structure, with a similar method which has been used in [6,7].

The paper has five sections and is organized as follows: Section 2 consists of the basic definitions, concepts, formulas, notations and results which will be used throughout the paper. Section 3 is devoted to parallelism, half parallelism and anti half parallelism of the distributions, which are naturally defined by the golden structure. We get equivalent statements related to parallelism of both of the distributions with respect to the fixed linear connection under the assumption that it is either the Levi-Civita connection or is not. We obtain a necessary and sufficient condition for each of the distributions to be half parallel with respect to the Schouten connection (respectively, the Vrănceanu connection). Also, we show that both of the distributions are always anti half parallel with respect to the Schouten and Vrănceanu connections. Section 4 is concerned with integrability of the golden structure and its associated distributions. We give necessary and sufficient conditions for the golden structure to be integrable. We obtain a condition for the Vrănceanu connection to be symmetric. We prove that the golden structure is integrable in the case that one of the Schouten and Vrănceanu connections is symmetric. By comparing the fixed linear connection with both the Schouten connection and the Vrănceanu connection, we obtain some results on integrability of both of the distributions and their leaves in the event that it is the Levi-Civita connection. The last section deals with the notion of geodesicity on golden manifolds in terms of the Schouten and Vrănceanu connections. We find a necessary and sufficient condition for a curve on a golden manifold to be a geodesic with respect to the Schouten connection (respectively, the Vrănceanu connection).

\section{Preliminaries}

This section presents some fundamental definitions, concepts, formulas, notations and results that will be needed in the remainder of the paper.

Let $\Phi$ be a golden structure on a $C^{\infty}$-differentiable real manifold $M$. We put

$$
r=\frac{1}{\sqrt{5}}((\phi-1) I+\Phi) \text { and } s=\frac{1}{\sqrt{5}}(\phi I-\Phi),
$$

where $\phi$ and $1-\phi$ are the roots of the algebraic equation $x^{2}-x-1=0$. Then we can easily verify that

$$
r+s=I, r^{2}=r, s^{2}=s, r s=0, s r=0,
$$

i.e., $r$ and $s$ are projection operators splitting the tangent bundle $T M$ into two complementary parts. Thus, the projection operators $r$ and $s$ define two globally complementary distributions of the tangent bundle $T M$. Furthermore, the golden structure $\Phi$ takes the form

$$
\Phi=\phi r+(1-\phi) s
$$


Let $R$ and $S$ be the distributions corresponding to the projection operators $r$ and $s$, respectively such that

$$
R=\bigcup_{p \in M} R_{p}, R_{p}=\left\{X_{p} \in T_{p} M: \Phi X_{p}=\phi X_{p}\right\}
$$

and

$$
S=\bigcup_{p \in M} S_{p}, S_{p}=\left\{X_{p} \in T_{p} M: \Phi X_{p}=(1-\phi) X_{p}\right\} .
$$

That is, the tangent bundle $T M$ has the decomposition $T M=R \oplus S$. Conversely, we assume that there exist two complementary distributions $R$ and $S$ on $M$. Then we can define a golden structure $\Phi$ on $M$ by putting

$$
\Phi=\phi r+(1-\phi) s,
$$

where $r$ and $s$ are the projection operators corresponding to the distributions $R$ and $S$, respectively. This expression of the golden structure $\Phi$ means that the following relations hold:

$$
\Phi r=r \Phi=\phi r
$$

and

$$
\Phi s=s \Phi=(1-\phi) s .
$$

Let $\nabla$ be an arbitrary fixed linear connection on $M$. Then $\nabla$ is a bilinear map

$$
\nabla: \Gamma(T M) \times \Gamma(T M) \longrightarrow \Gamma(T M),
$$

defined by the rule

$$
(X, Y) \longrightarrow \nabla_{X} Y
$$

and satisfying the following two properties:

(a) $\nabla_{f X} Y=f \nabla_{X} Y$,

(b) $\nabla_{X}(f Y)=f \nabla_{X} Y+(X f) Y$

for an arbitrary differentiable function $f \in C^{\infty}(M)$ and any vector fields $X, Y \in \Gamma(T M)$. The operator $\nabla_{X}$ is called the covariant differentiation with respect to $X$. The torsion tensor $\tau(\nabla)$ of the linear connection $\nabla$ is given by

$$
\tau(\nabla)(X, Y)=\nabla_{X} Y-\nabla_{Y} X-[X, Y]
$$

for any vector fields $X, Y \in \Gamma(T M)$. The linear connection is symmetric if its torsion tensor $\tau(\nabla)$ vanishes identically. The linear connection is called a metric connection if there is a Riemannian metric $g$ on $M$ such that $g$ is parallel. It is well known that on an arbitrary Riemannian manifold, there exists one and only one symmetric metric connection, called the Levi-Civita connection.

The covariant derivative of the golden structure $\Phi$ is defined by

$$
\left(\nabla_{X} \Phi\right) Y=\nabla_{X} \Phi Y-\Phi \nabla_{X} Y
$$

for any vector fields $X, Y \in \Gamma(T M)$. We say that the golden structure $\Phi$ is parallel with respect to the linear connection $\nabla$ if its covariant derivative $\nabla \Phi$ is identically zero. The same definitions apply to the projection operators $r$ and $s$.

We define two maps $\stackrel{S c}{\nabla}$ and $\stackrel{V}{\nabla}$ by the rules

$$
\stackrel{S c}{\nabla}_{X} Y=r\left(\nabla_{X} r Y\right)+s\left(\nabla_{X} s Y\right)
$$

and

$$
\nabla_{X} Y=r\left(\nabla_{r X} r Y\right)+s\left(\nabla_{s X} s Y\right)+r[s X, r Y]+s[r X, s Y]
$$

for any vector fields $X, Y \in \Gamma(T M)$, respectively. $\stackrel{S c}{\nabla}$ and $\stackrel{V}{\nabla}$ are called Schouten connection and Vrănceanu connection, respectively $[8,9]$.

It is not difficult to show that each of the Schouten and Vrănceanu connections is a linear connection on $M$. 


\section{Parallelism, Half Parallelism and Anti Half Parallelism on Golden Manifolds}

In this section, we discuss parallelism, half parallelism and anti half parallelism of the distributions associated with the golden structure $\Phi$.

As it is well known, a distribution $D$ on $M$ is called parallel with respect to the linear connection $\nabla$ if the vector field $\nabla_{X} Y$ lies in $\Gamma(D)$ for any vector fields $X \in \Gamma(T M)$ and $Y \in \Gamma(D)$.

The notions of half parallelism and anti half parallelism have been introduced for the distributions defined on Lagrangian $F\left(a_{1}, a_{2}, \ldots, a_{n}\right)$-structure manifold in [7]. Now, we apply similar definitions to the distributions $R$ and $S$. We put

$$
(\Delta \Phi)(X, Y)=\Phi \nabla_{X} Y-\Phi \nabla_{Y} X-\nabla_{\Phi X} Y+\nabla_{Y} \Phi X
$$

for any vector fields $X, Y \in \Gamma(T M)$. Then we have the following definitions:

Definition 3.1. The distribution $R$ on $M$ is called half parallel with respect to the linear connection $\nabla$ if the vector field $(\Delta \Phi)(X, Y)$ belongs to $\Gamma(R)$ for any vector fields $X \in \Gamma(R)$ and $Y \in \Gamma(T M)$.

Definition 3.2. The distribution $R$ on $M$ is called anti half parallel with respect to the linear connection $\nabla$ if the vector field $(\Delta \Phi)(X, Y)$ pertains to $\Gamma(S)$ for any vector fields $X \in \Gamma(R)$ and $Y \in \Gamma(T M)$.

In the same manner, half parallelism and anti half parallelism of the distribution $S$ can be defined.

Proposition 3.1. [3] The following assertions are satisfied:

(a) Both of the distributions $R$ and $S$ are parallel with respect to the Schouten and Vrănceanu connections.

(b) Both of the projection operators $r$ and s are parallel with respect to the Schouten and Vrănceanu connections.

(c) The golden structure $\Phi$ is parallel with respect to the Schouten and Vrănceanu connections.

Proposition 3.2. The following assertions are equivalent:

(a) Both of the distributions $R$ and $S$ are parallel with respect to the linear connection $\nabla$.

(b) The golden structure $\Phi$ is parallel with respect to the linear connection $\nabla$.

(c) Both of the projection operators $r$ and s are parallel with respect to the linear connection $\nabla$.

Proof. Using the fact that $r+s=I$, we have

$$
\left(\nabla_{X} \Phi\right) Y=\nabla_{X} \Phi Y-\Phi \nabla_{X} Y=\nabla_{X} \Phi r Y+\nabla_{X} \Phi s Y-\Phi \nabla_{X} r Y-\Phi \nabla_{X} s Y
$$

for any vector fields $X, Y \in \Gamma(T M)$. Let us consider that both of the distributions $R$ and $S$ are parallel with respect to the linear connection $\nabla$. Then taking account of the fact that $\nabla$ is a linear connection, it is immediately obtained from (2.3) that we have

$$
\nabla \Phi=0,
$$

which proves $(\mathrm{a}) \Rightarrow(\mathrm{b})$. Taking into account the fact that $\nabla I=0$, it follows from (2.1) that

$$
\nabla r=-\nabla s=\frac{1}{\sqrt{5}} \nabla \Phi .
$$

If the golden structure $\Phi$ is parallel with respect to the linear connection $\nabla$, then we get

$$
\nabla r=\nabla s=0,
$$

from which we have $(\mathrm{b}) \Rightarrow(\mathrm{c})$. Providing that both of the projection operators $r$ and $s$ are parallel with respect to the linear connection $\nabla$, we obtain

$$
\nabla_{X} r Y=r \nabla_{X} Y \in \Gamma(R)
$$

and

$$
\nabla_{X} s Y=s \nabla_{X} Y \in \Gamma(S)
$$

for any vector fields $X, Y \in \Gamma(T M)$. Then it results from (3.3) and (3.4) that we have (c) $\Rightarrow(\mathrm{a})$. Therefore, the proof has been completed.

Theorem 3.1. The following assertions are equivalent:

(a) Both of the distributions $R$ and $S$ are parallel with respect to the linear connection $\nabla$. 
(b) The Schouten connection $\stackrel{S c}{\nabla}$ is equal to the linear connection $\nabla$.

Proof. If both of the distributions $R$ and $S$ are parallel with respect to the linear connection $\nabla$, then for any vector fields $X, Y \in \Gamma(T M)$, we get by a direct calculation that

$$
\stackrel{S c}{\nabla}_{X} Y=r\left(\nabla_{X} r Y\right)+s\left(\nabla_{X} s Y\right)=\nabla_{X} r Y+\nabla_{X} s Y=\nabla_{X} Y,
$$

which shows $(\mathrm{a}) \Rightarrow(\mathrm{b})$. We assume that $\stackrel{S c}{\nabla}$ and $\nabla$ are equal. By virtue of (2.13), we obtain

$$
\nabla_{X} r Y=\stackrel{S c}{\nabla}_{X} r Y=r\left(\nabla_{X} r^{2} Y\right)+s\left(\nabla_{X} s r Y\right)=r \nabla_{X} r Y \in \Gamma(R)
$$

and

$$
\nabla_{X} s Y=\stackrel{S c}{\nabla}_{X} s Y=r\left(\nabla_{X} r s Y\right)+s\left(\nabla_{X} s^{2} Y\right)=s\left(\nabla_{X} s Y\right) \in \Gamma(S)
$$

for any vector fields $X, Y \in \Gamma(T M)$. Then it follows from (3.6) and (3.7) that we have (b) $\Rightarrow(a)$. Hence, the proof has been finished.

Corollary 3.1. The following assertions are equivalent:

(a) Both of the distributions $R$ and $S$ are parallel with respect to the linear connection $\nabla$.

(b) The golden structure $\Phi$ is parallel with respect to the linear connection $\nabla$.

(c) Both of the projection operators $r$ and s are parallel with respect to the linear connection $\nabla$.

(d) The Schouten connection $\stackrel{S c}{\nabla}$ is equal to the linear connection $\nabla$.

Proof. Combining Proposition 3.2 and Theorem 3.1, the proof is obvious.

Proposition 3.3. Let $\nabla$ be the Levi-Civita connection on a golden Riemannian manifold $(M, g, \Phi)$. The following assertions are equivalent:

(a) The distribution $R$ is parallel with respect to the Levi-Civita connection $\nabla$.

(b) The distribution $S$ is parallel with respect to the Levi-Civita connection $\nabla$.

Proof. Using the fact that $\nabla g=0$, we get

$$
g\left(\nabla_{X} r Y, s Z\right)=-g\left(r Y, \nabla_{X} s Z\right)
$$

for any vector fields $X, Y, Z \in \Gamma(T M)$. Then (3.8) states that (a) is equivalent to (b).

Theorem 3.2. Let $\nabla$ be the Levi-Civita connection on a golden Riemannian manifold $(M, g, \Phi)$. The Schouten connection $\stackrel{S c}{\nabla}$ is equal to the Levi-Civita connection $\nabla$ if and only if one of the distributions $R$ and $S$ is parallel with respect to the Levi-Civita connection $\nabla$.

Proof. Applying Proposition 3.3 to Theorem 3.1, the proof has been shown.

Theorem 3.3. The following assertions are satisfied:

(a) If the Vrănceanu connection $V$ is equal to the linear connection $\nabla$, then both of the distributions $R$ and $S$ are parallel with respect to the linear connection $\nabla$.

(b) Let us suppose that the linear connection $\nabla$ is symmetric. If both of the distributions $R$ and $S$ are parallel with respect to the linear connection $\nabla$, then the Vrănceanu connection $V$ is equal to the linear connection $\nabla$.

Proof. We assume that the Vrănceanu connection $\stackrel{V}{\nabla}$ is equal to the linear connection $\nabla$. By a straightforward computation, we obtain from (2.14) that

$$
\nabla_{X} r Y=\stackrel{V}{\nabla}_{X} r Y=r\left(\nabla_{r X} r Y\right)+r[s X, r Y] \in \Gamma(R)
$$


and

$$
\nabla_{X} s Y=\nabla_{X} s Y=s\left(\nabla_{s X} s Y\right)+s[r X, s Y] \in \Gamma(S)
$$

for any vector fields $X, Y \in \Gamma(T M)$. Hence, (a) follows from (3.9) and (3.10). If the linear connection $\nabla$ is symmetric, the expression of the Vrănceanu connection $\nabla$ in (2.14) takes the form

$$
\nabla_{X} Y=r\left(\nabla_{X} r Y\right)+s\left(\nabla_{X} s Y\right)-r\left(\nabla_{r Y} s X\right)-s\left(\nabla_{s Y} r X\right)
$$

for any vector fields $X, Y \in \Gamma(T M)$. By the assumption that both of the distributions $R$ and $S$ are parallel with respect to the linear connection $\nabla$, it is easy to see that we have (b).

Theorem 3.4. Let $\nabla$ be the Levi-Civita connection on a golden Riemannian manifold $(M, g, \Phi)$. The Vrănceanu connection $\nabla$ is equal to the Levi-Civita connection $\nabla$ if and only if one of the distributions $R$ and $S$ is parallel with respect to the Levi-Civita connection $\nabla$.

Proof. The proof is clear from Proposition 3.3 and Theorem 3.3.

Corollary 3.2. Let $\nabla$ be the Levi-Civita connection on a golden Riemannian manifold $(M, g, \Phi)$. The following assertions are equivalent:

(a) One of the distributions $R$ and $S$ is parallel with respect to the Levi-Civita connection $\nabla$.

(b) Both the Schouten connection $\stackrel{S c}{\nabla}$ and the Vrănceanu connection $\stackrel{V}{\nabla}$ are equal to the Levi-Civita connection $\nabla$.

Proof. The proof is trivial from Theorem 3.2 and Theorem 3.4.

Theorem 3.5. The distribution $R$ is half parallel with respect to the Schouten connection $\stackrel{S c}{\nabla}$ if and only if

$$
\nabla_{X} s Y \in \Gamma(R)
$$

for any vector fields $X \in \Gamma(R)$ and $Y \in \Gamma(T M)$.

Proof. Taking into consideration (3.1), we have

$$
s(\stackrel{S c}{\Delta} \Phi)(X, Y)=s \Phi \nabla^{S c} \nabla_{X} Y-s \Phi \stackrel{S c}{\nabla}_{Y} X-s \stackrel{S c}{\nabla}_{\Phi X} Y+s \stackrel{S c}{\nabla_{Y}} \Phi X
$$

for any vector fields $X \in \Gamma(R)$ and $Y \in \Gamma(T M)$. Applying (2.4) and (2.8) in (3.13), by a simple computation, we obtain from (2.13) that

$$
s(\stackrel{S c}{\Delta} \Phi)(X, Y)=(1-2 \phi) s\left(\stackrel{S c}{\nabla}_{X} Y-\stackrel{S c}{\nabla}_{Y} X\right)=-\sqrt{5} s\left(\nabla_{X} s Y\right) .
$$

Then (3.14) means that half parallelism of the distribution $R$ with respect to the Schouten connection $\stackrel{S c}{\nabla}$ is equivalent to the condition that

$$
\nabla_{X} s Y \in \Gamma(R)
$$

for any vector fields $X \in \Gamma(R)$ and $Y \in \Gamma(T M)$.

Theorem 3.6. The distribution $S$ is half parallel with respect to the Schouten connection $\stackrel{S c}{\nabla}$ if and only if

$$
\nabla_{X} r Y \in \Gamma(S)
$$

for any vector fields $X \in \Gamma(S)$ and $Y \in \Gamma(T M)$.

Proof. The proof can be shown in a manner similar to that of Theorem 3.5.

Theorem 3.7. The distribution $R$ is half parallel with respect to the Vrănceanu connection $\stackrel{V}{\nabla}$ if and only if

$$
[X, s Y] \in \Gamma(R)
$$

for any vector fields $X \in \Gamma(R)$ and $Y \in \Gamma(T M)$. 
Proof. Let $X \in \Gamma(R)$ and $Y \in \Gamma(T M)$. Taking into account (3.1), we have

$$
s(\stackrel{V}{\Delta} \Phi)(X, Y)=s \Phi \nabla_{X} Y-s \Phi \nabla_{Y} X-s \nabla_{\Phi X} Y+s \stackrel{V}{\nabla} \nabla_{Y} \Phi X .
$$

Using the explicit expression of the Vrănceanu connection in (2.14), by a direct calculation, we get from (2.4) and (2.8) that

$$
s\left(\begin{array}{l}
V \\
\Delta
\end{array}\right)(X, Y)=(1-2 \phi) s\left(\nabla_{X} Y-\nabla_{Y} X\right)=-\sqrt{5} s[X, s Y]
$$

which implies that $R$ is half parallel with respect to the Vrănceanu connection $V$ if and only if the vector field $[X, s Y]$ lies in $\Gamma(R)$ for any vector fields $X \in \Gamma(R)$ and $Y \in \Gamma(T M)$.

Theorem 3.8. The distribution $S$ is half parallel with respect to the Vrănceanu connection $V$ if and only if

$$
[X, r Y] \in \Gamma(S)
$$

for any vector fields $X \in \Gamma(S)$ and $Y \in \Gamma(T M)$.

Proof. The proof is similar to that of Theorem 3.7.

Proposition 3.4. Both of the distributions $R$ and $S$ are anti half parallel with respect to the linear connection $\nabla$.

Proof. From (3.1), we have

$$
r(\Delta \Phi)(X, Y)=r \Phi \nabla_{X} Y-r \Phi \nabla_{Y} X-r \nabla_{\Phi X} Y+r \nabla_{Y} \Phi X
$$

for any vector fields $X \in \Gamma(R)$ and $Y \in \Gamma(T M)$. As $\nabla$ is a linear connection, it is clear from (2.4) and (2.7) that

$$
r(\Delta \Phi)(X, Y)=0
$$

for any vector fields $X \in \Gamma(R)$ and $Y \in \Gamma(T M)$. Then (3.22) implies that the distribution $R$ is anti half parallel with respect to the linear connection $\nabla$. Analogously, it can be shown that the distribution $S$ is anti half parallel with respect to the linear connection $\nabla$.

Taking into consideration that $\nabla$ is an arbitrary linear connection on $M$, it also states that both of the distributions $R$ and $S$ are anti half parallel with respect to the Schouten and Vrănceanu connections by virtue of Proposition 3.4.

\section{Integrability on Golden Manifolds}

The aim of this section is to give some facts on integrability of the golden structure $\Phi$ and its associated distributions.

Let $\varphi$ be a tensor field of type $(1,1)$ on $M$. Recall that

$$
N_{\varphi}=\frac{1}{2}[\varphi, \varphi]_{F N}
$$

where $[., .]_{F N}$ is the Frölicher-Nijenhuis bracket of vector-valued forms. It has a key role in measuring integrability of geometric structures on differentiable manifolds of class $C^{\infty}$. Moreover, we have the following relations:

$$
[I, \varphi]_{F N}=0
$$

and

$$
N_{\varphi_{1}+\varphi_{2}}=N_{\varphi_{1}}+N_{\varphi_{2}}+\left[\varphi_{1}, \varphi_{2}\right]_{F N}
$$

where $\varphi_{1}$ and $\varphi_{2}$ are tensor fields of type $(1,1)$ on $M$.

As it is well-known, the golden structure $\Phi$ is a tensor field of type $(1,1)$ on $M$ satisfying (1.2). The Nijenhuis tensor of the golden structure $\Phi$ is given by

$$
N_{\Phi}(X, Y)=[\Phi X, \Phi Y]-\Phi[\Phi X, Y]-\Phi[X, \Phi Y]+\Phi[X, Y]+[X, Y]
$$


for any vector fields $X, Y \in \Gamma(T M)$. It has the following basic properties:

$$
N_{\lambda \Phi}=\lambda^{2} N_{\Phi}
$$

and

$$
N_{\Phi+I}=N_{\Phi^{2}}=N_{\Phi}=N_{\Phi^{-1}},
$$

where $\lambda$ is a real constant and $I$ is the identity $(1,1)$-tensor field on $M$.

Lemma 4.1. The following relations are valid:

$$
\begin{aligned}
& N_{r}(X, Y)=N_{s}(X, Y), \\
& N_{r}(X, Y)=-\frac{1}{2}[r, s]_{F N}(X, Y), \\
& N_{r}(X, Y)=\frac{1}{5} N_{\Phi}(X, Y), \\
& N_{r}(X, Y)=s[r X, r Y]+r[s X, s Y]
\end{aligned}
$$

for arbitrary vector fields $X, Y \in \Gamma(T M)$.

Proof. Recall that the Nijenhuis tensors of the projection operators $r$ and $s$ are given by

$$
N_{r}=\frac{1}{2}[r, r]_{F N}
$$

and

$$
N_{s}=\frac{1}{2}[s, s]_{F N},
$$

respectively. Using the fact that $r+s=I$, we get

$$
N_{r}=\frac{1}{2}[r, r]_{F N}=\frac{1}{2}[I-s, I-s]_{F N}=\frac{1}{2}[I, I]_{F N}-\frac{1}{2}[s, I]_{F N}-\frac{1}{2}[I, s]_{F N}+\frac{1}{2}[s, s]_{F N}
$$

by virtue of the bilinearity of the Frölicher-Nijenhuis bracket. From (4.2), we have

$$
N_{r}=\frac{1}{2}[s, s]_{F N},
$$

which immediately implies (4.7). Taking into account the fact that $r+s=I$ and the bilinearity of the FrölicherNijenhuis bracket, we get from (4.2) that

$$
\frac{1}{2}[r, s]_{F N}=\frac{1}{2}[r, I]_{F N}-\frac{1}{2}[r, r]_{F N}=-\frac{1}{2}[r, r]_{F N}=-N_{r},
$$

which proves (4.8). Notice that the golden structure $\Phi$ has the form $\Phi=\phi r+(1-\phi) s$ in terms of the projection operators $r$ and $s$. From (4.3), the Nijenhuis tensor of the golden structure $\Phi$ is given

$$
N_{\Phi}=N_{\phi r}+N_{(1-\phi) s}+[\phi r,(1-\phi) s]_{F N} .
$$

By a straightforward calculation, we obtain from (4.5) and (4.15) that

$$
N_{\Phi}=\phi^{2} N_{r}+(1-\phi)^{2} N_{s}+\phi(1-\phi)[r, s]_{F N}=\phi^{2} N_{r}+(1-\phi)^{2} N_{r}-2 \phi(1-\phi) N_{r}=5 N_{r},
$$

from which we have (4.9). As it is well-known, the Nijenhuis tensor of the projection operator $r$ is also given by

$$
N_{r}(X, Y)=[r X, r Y]-r[r X, Y]-r[X, r Y]+r[X, Y]
$$

for any vector fields $X, Y \in \Gamma(T M)$. By reason of the fact that $r+s=I$, (4.18) takes the form

$$
N_{r}(X, Y)=r[r X, r Y]+s[r X, r Y]+r[s X, Y]-r[X, r Y] .
$$

Using again the fact that $r+s=I$ in (4.19), it is easy to deduce that

$$
N_{r}(X, Y)=s[r X, r Y]+r[s X, s Y],
$$

which is the evidence of (4.10). Hence, the proof has been completed. 
Lemma 4.1 allows us to establish a close relationship between integrability of the golden structure $\Phi$ and its associated distributions $R$ and $S$. Therefore, we have the following theorem:

Theorem 4.1. The golden structure $\Phi$ is integrable if and only if both of the distributions $R$ and $S$ are integrable.

Proof. As it is well known, the distribution $R$ is integrable if and only if $s[r X, r Y]=0$ and the distribution $S$ is integrable if and only if $r[s X, s Y]=0$ for any vector fields $X, Y \in \Gamma(T M)$. Recall also that the golden structure $\Phi$ is integrable if its Nijenhuis tensor $N_{\Phi}$ vanishes identically. It follows from (4.9) and (4.10) that

$$
\frac{1}{5} N_{\Phi}(X, Y)=s[r X, r Y]+r[s X, s Y]
$$

for any vector fields $X, Y \in \Gamma(T M)$. As a consequence of (4.21), the proof is clear.

M. C. Crâşmăreanu and C. E. Hreţcanu have just proved the necessity of Theorem 4.1 as a proposition in [3]. We have also demonstrated that the golden structure $\Phi$ is integrable if both of the distributions $R$ and $S$ are integrable. In other words, the proposition has been developed by us.

Now, we give another theorem related to integrability of the golden structure $\Phi$ by means of Lemma 4.1.

Theorem 4.2. The golden structure $\Phi$ is integrable if and only if the Frölicher-Nijenhuis bracket of the projection operators $r$ and $s$ vanishes identically.

Proof. Taking account that integrability of the golden structure $\Phi$ is equivalent to that its Nijenhuis tensor is identically zero, the proof has been obtained from (4.8) and (4.9).

Corollary 4.1. The following assertions are equivalent:

(a) The golden structure $\Phi$ is integrable.

(b) Both of the distributions $R$ and $S$ are integrable.

(c) The Frölicher-Nijenhuis bracket of the projection operators $r$ and $s$ vanishes identically.

Proof. Combining Theorem 4.1 and Theorem 4.2, the proof is clear.

Theorem 4.3. If the linear connection $\nabla$ is symmetric and the golden structure $\Phi$ is integrable, then the Vrănceanu connection $\nabla$ is symmetric.

Proof. We denote by $\tau\left(\frac{V}{\nabla}\right)$ the torsion tensor of the Vrănceanu connection $\nabla$. It is given by

$$
\tau(\stackrel{V}{\nabla})(X, Y)=\stackrel{V}{\nabla}_{X} Y-\stackrel{V}{\nabla}_{Y} X-[X, Y]
$$

for any vector fields $X, Y \in \Gamma(T M)$. Applying the projection operator $r$ from the left hand side to (4.22), we obtain

$$
r \tau(\stackrel{V}{\nabla})(X, Y)=r\left(\nabla_{r X} r Y-\nabla_{r Y} r X\right)+r[s X, r Y]-r[s Y, r X]-r[X, Y] .
$$

Since the linear connection $\nabla$ is symmetric, (4.23) takes the form

$$
r \tau(\stackrel{V}{\nabla})(X, Y)=r[r X, r Y]+r[s X, r Y]-r[s Y, r X]-r[X, Y] .
$$

Taking account of the bilinearity and the anti-commutativity of the Lie bracket [., .], it follows from the relations in (2.2) that

$$
r \tau(\nabla)(X, Y)=r[s Y, s X]
$$

Similarly, we find

$$
s \tau(\stackrel{V}{\nabla})(X, Y)=s[r Y, r X]
$$


Taking into account that the golden structure $\Phi$ is integrable, both of the distributions $R$ and $S$ are integrable by virtue of Theorem 4.1 . That is,

$$
r[s Y, s X]=s[r Y, r X]=0
$$

for any vector fields $X, Y \in \Gamma(T M)$. Then we obtain from (4.25) and (4.26) that

$$
r \tau(\stackrel{V}{\nabla})(X, Y)=s \tau(\stackrel{V}{\nabla})(X, Y)=0 .
$$

Thus, we have

$$
\tau(\stackrel{V}{\nabla})(X, Y)=r \tau(\stackrel{V}{\nabla})(X, Y)+s \tau(\stackrel{V}{\nabla})(X, Y)=0,
$$

i.e., $\tau(\stackrel{V}{\nabla})$ is identically zero.

Theorem 4.4. If one of the Schouten and Vrănceanu connections is symmetric, then the golden structure $\Phi$ is integrable.

Proof. If the Schouten connection is symmetric, we have

$$
\stackrel{S c}{\nabla}{ }_{X} Y-\stackrel{S c}{\nabla} Y=[X, Y]
$$

for any vector fields $X, Y \in \Gamma(T M)$. Substituting $X$ by $r X$ and $Y$ by $r Y$ in (4.30), we get

$$
s[r X, r Y]=s \stackrel{S c}{\nabla}_{r X} r Y-s \stackrel{S c}{\nabla}_{r Y} r X .
$$

Taking into account that the distribution $R$ is parallel with respect to the Schouten connection, we obtain from (a) of Proposition 3.1 that

$$
s[r X, r Y]=0 .
$$

Similary, it can be shown that

$$
r[s X, s Y]=0
$$

for any vector fields $X, Y \in \Gamma(T M)$. Then (4.32) and (4.33) imply that the distributions $R$ and $S$ are integrable, respectively. Thus, the golden structure $\Phi$ is integrable by virtue of Theorem 4.1. If the Vrănceanu connection is symmetric, in the same manner, it can be easily shown that the golden structure $\Phi$ is integrable.

Theorem 4.5. Let $\nabla$ be the Levi-Civita connection on a golden Riemannian manifold $(M, g, \Phi)$. The Schouten connection $\stackrel{S c}{\nabla}$ is equal to the Levi-Civita connection $\nabla$ if and only if both of the distributions $R$ and $S$ are integrable and their leaves are totally geodesic in $M$.

Proof. We assume that the Schouten connection $\stackrel{S c}{\nabla}$ is equal to the Levi-Civita connection $\nabla$. Since the Levi-Civita connection $\nabla$ is symmetric, it follows from (a) of Proposition 3.1 that

$$
[r X, r Y]=\nabla_{r X} r Y-\nabla_{r Y} r X=\stackrel{S c}{\nabla}_{r X} r Y-\stackrel{S c}{\nabla}_{r Y} r X \in \Gamma(R)
$$

and

$$
[s X, s Y]=\nabla_{s X} s Y-\nabla_{s Y} s X=\stackrel{S c}{\nabla}_{s X} s Y-\stackrel{S c}{\nabla}_{s Y} s X \in \Gamma(S)
$$

for any vector fields $X, Y \in \Gamma(T M)$. Then it results from (4.34) and (4.35) that the distributions $R$ and $S$ are integrable, respectively. Let $M^{R}$ be a leaf of the distribution $R$. Taking into account that the distribution $R$ is always parallel with respect to the Schouten connection $\stackrel{S c}{\nabla}$, we obtain from the definition of the leaf $M^{R}$ that

$$
\stackrel{S c}{\nabla}_{X} Y \in \Gamma\left(M^{R}\right)
$$

for any vector fields $X, Y \in \Gamma\left(M^{R}\right)$. On the other hand, from Gauss formula, we have

$$
\stackrel{S c}{\nabla}_{X} Y=\nabla_{X} Y=\nabla_{X}^{R} Y+h^{R}(X, Y)
$$


for any vector fields $X, Y \in \Gamma\left(M^{R}\right)$, where $\nabla^{R}$ is the Levi-Civita connection on $M^{R}, h^{R}$ is the second fundamental form of the immersion of $M^{R}$ in $M$ and $h^{R}(X, Y) \in \Gamma(S)$. Combining (4.36) and (4.37), then we get

$$
h^{R}=0,
$$

which implies that $M^{R}$ is totally geodesic in $M$. Similarly, it can be shown that each leaf of the distribution $S$ is totally geodesic in $M$. Conversely, we suppose that both of the distributions $R$ and $S$ are integrable and their leaves are totally geodesic in $M$. By virtue of Gauss formula, we obtain

$$
\nabla_{r X} r Y \in \Gamma(R)
$$

and

$$
\nabla_{s X} s Y \in \Gamma(S)
$$

for any vector fields $X, Y \in \Gamma(T M)$. Since the Riemannian metric $g$ is parallel with respect to the Levi-Civita connection $\nabla$, it follows from (4.39) and (4.40) that

$$
g\left(\nabla_{X} r Y, s Z\right)=g\left(\nabla_{s X} r Y, s Z\right)=-g\left(r Y, \nabla_{s X} s Z\right)=0
$$

and

$$
g\left(\nabla_{X} s Y, r Z\right)=g\left(\nabla_{r X} s Y, r Z\right)=-g\left(s Y, \nabla_{r X} r Z\right)=0
$$

for any vector fields $X, Y, Z \in \Gamma(T M)$, respectively. Then (4.41) and (4.42) imply that the distributions $R$ and $S$ are parallel with respect to the Levi-Civita connection $\nabla$, respectively. From Theorem 3.1, we get

$$
\stackrel{S c}{\nabla}=\nabla \text {. }
$$

Thus, the proof has been completed.

Theorem 4.6. Let $\nabla$ be the Levi-Civita connection on a golden Riemannian manifold $(M, g, \Phi)$. The Vrănceanu connection $\nabla$ is equal to the Levi-Civita connection $\nabla$ if and only if both of the distributions $R$ and $S$ are integrable and their leaves are totally geodesic in $M$.

Proof. The proof can be demonstrated in a method similar to that of Theorem 4.5.

Corollary 4.2. Let $\nabla$ be the Levi-Civita connection on a golden Riemannian manifold $(M, g, \Phi)$. The following assertions are equivalent:

(a) Both of the distributions $R$ and $S$ are integrable and their leaves are totally geodesic in $M$.

(b) Both the Schouten connection $\stackrel{S c}{\nabla}$ and the Vrănceanu connection $\stackrel{V}{\nabla}$ are equal to the Levi-Civita connection $\nabla$.

Proof. The proof is clear from Theorem 4.5 and Theorem 4.6.

\section{Geodesics on Golden Manifolds}

This is a short section whose purpose is briefly to mention the concept of geodesicity on golden manifolds with regard to the Schouten and Vrănceanu connections.

We consider a curve $\alpha$ on the golden manifold $(M, \Phi)$. Let $T$ be tangent vector field of the curve $\alpha$. The curve $\alpha$ is called a geodesic with respect to the linear connection $\nabla$ if $\nabla_{T} T=0$.

Theorem 5.1. The curve $\alpha$ is a geodesic with respect to the Schouten connection $\stackrel{S c}{\nabla}$ if and only if

$$
\nabla_{T} r T \in \Gamma(S) \text { and } \nabla_{T} s T \in \Gamma(R) .
$$

Proof. By the definition of the Schouten connection in (2.13), we have

$$
\stackrel{S c}{\nabla}_{T} T=r\left(\nabla_{T} r T\right)+s\left(\nabla_{T} s T\right) .
$$


If the curve $\alpha$ is a geodesic with respect to the Schouten connection $\stackrel{S c}{\nabla}$, we get

$$
r\left(\nabla_{T} r T\right)+s\left(\nabla_{T} s T\right)=0,
$$

which shows

$$
\nabla_{T} r T \in \Gamma(S) \text { and } \nabla_{T} s T \in \Gamma(R) .
$$

Conversely, if the relations are valid in (5.1), it is quite obvious that

$$
\stackrel{S c}{\nabla_{T} T}=0
$$

that is, the curve $\alpha$ is a geodesic with respect to the Schouten connection.

Theorem 5.2. The curve $\alpha$ is a geodesic with respect to the Vrănceanu connection $\stackrel{V}{\nabla}$ if and only if

$$
\nabla_{r T} r T+[s T, r T] \in \Gamma(S) \text { and } \nabla_{s T} s T+[r T, s T] \in \Gamma(R) .
$$

Proof. In view of (2.14), we have

$$
\stackrel{V}{\nabla}_{T} T=r\left(\nabla_{r T} r T\right)+s\left(\nabla_{s T} s T\right)+r[s T, r T]+s[r T, s T] .
$$

Provided that the relations are satisfied in (5.6), then it is clear that

$$
V_{T}^{V} T=0,
$$

i.e., the curve $\alpha$ is a geodesic with respect to the Vrănceanu connection. Now, we assume that the curve $\alpha$ is a geodesic with respect to the Vrănceanu connection $\nabla$. Then (5.7) states that

$$
\nabla_{r T} r T+[s T, r T] \in \Gamma(S) \text { and } \nabla_{s T} s T+[r T, s T] \in \Gamma(R) .
$$

Therefore, the proof has been completed.

\section{Acknowledgments}

The authors are very much thankful to the referees for their valuable comments and suggestions.

\section{References}

[1] Goldberg, S. I. and Yano, K., Polynomial Structures on Manifolds. Kodai Math. Sem. Rep. 22 (1970), no. 2, $199-218$.

[2] Hreţcanu, C. E., Submanifolds in Riemannian Manifolds with Golden Structure. Acta Math. Acad. Paedagog. Nyházi. (N.S.) 24 (2008), no. 1, 3-4.

[3] Crâşmăreanu, M. C. and Hreţcanu, C. E., Golden Differential Geometry. Chaos Soliton. Fract. 38 (2008), no. 5, $1229-1238$.

[4] Hreţcanu, C.E. and Crâşmăreanu, M. C., On Some Invariant Submanifolds in a Riemannian Manifold with Golden Structure. An. Ştiinţ. Univ. Al. I. Cuza Iaşi. Mat. (N.S.) 53 (2007), suppl. 1, 199-211.

[5] Hrețcanu, C. E. and Crâşmăreanu, M. C., Applications of the Golden Ratio on Riemannian Manifolds. Turk. J. Math. 33 (2009), no. 2, 179-191.

[6] Ianuş, S., Some Almost Product Structures on Manifolds with Linear Connection. Kodai Math. Sem. Rep. 23 (1971), no. 3 , 305-310.

[7] Das, L. S., Nikić, J. and Nivas, R., Parallelism of Distributions and Geodesics on $F\left(a_{1}, a_{2}, \ldots, a_{n}\right)$-structure Lagrangian Manifolds. Differ. Geom. Dyn. Syst. 8 (2006), no. 1, 82-89.

[8] Bejancu, A. and Farran, H. D., Foliations and Geometric Structures. Springer, Amsterdam, 2006.

[9] Ianus, S., Sur les Structures Presque Produit des Varietes a Connection Lineairei. C. R. Acad. Sci. 272 (1971), no. 11, $734-735$. 


\section{Affiliations}

MUSTAFA GÖK

AdDRESS: Inonü University, Dept. of Mathematics, 44280, Malatya-Turkey. E-MAIL: mustafa.gok@email.com

ORCID ID:0000-0001-6346-0758

SADIK KELEŞ

ADDRESS: Inonü University, Dept. of Mathematics, 44280, Malatya-Turkey. E-MAIL: sadik.keles@inonu.edu.tr

ORCID ID:0000-0003-3981-2092

EROL KILIÇ

ADDRESS: Inonü University, Dept. of Mathematics, 44280, Malatya-Turkey.

E-MAIL: erol.kilic@inonu.edu.tr

ORCID ID:0000-0001-7536-0404 